\title{
Orbital-free ab initio molecular dynamics study of the static structure and dynamic properties of the free liq- uid surface of Sn.
}

\author{
B. G. del Rio ${ }^{1, *}$ and L. E. González ${ }^{1}$ \\ ${ }^{1}$ Departamento de Fisica Teórica, Facultad de Ciencias, Universidad de Valladolid, Val- \\ ladolid, SPAIN
}

\begin{abstract}
We report results of an orbital-free ab initio molecular dynamics (OF-AIMD) study of the free liquid surface (FLS) of Sn at $1000 \mathrm{~K}$ and $600 \mathrm{~K}$. A key ingredient in the OF-AIMD method is the local pseudopotential describing the ions-valence electrons interaction. We have used a force-matching method to derive a local pseudopotential suitable to account for the variation of the forces from the bulk to the FLS. We obtain very good results for structural properties, such as the reflectivity, including the characteristic shoulder it presents in $\mathrm{x}$-ray experiments. Moreover we have been able to study ab initio for the first time the evolution in some dynamical properties as we move from the central region, where the system behaves like the bulk liquid, to the FLS.
\end{abstract}

\section{Introduction.}

Molecular dynamics (MD) simulations have become an essential technique in the study of a wide variety of condensed matter systems, and during the last three decades there has been an increasing amount of systems studied by using ab initio molecular dynamics (AIMD) methods based on density functional theory (DFT) [1]. Most AIMD methods are based on the Kohn-Sham (KS) orbital representation of DFT (KS-AIMD methods) which, although very accurate, imposes high computational demands that severely limit the size of the systems to be studied as well as the extent of the simulation times. Nevertheless, these constraints may be drastically reduced by the orbital-free ab-initio molecular dynamics (OF-AIMD) simulation method [2], whose basic variable is the total valence electronic density instead of the electronic orbitals of the KS-AIMD formulation. The OF-AIMD method substantially reduces the number of variables needed to describe the electronic state and therefore allows to study larger systems for longer simulation times. However, this improvement is achieved at the price of resorting to an approximate description of the electronic kinetic energy, and then, in principle, the OF-AIMD approach is less accurate than the KS-AIMD method. Nevertheless, despite this caveat, the OF-AIMD has provided good descriptions for several static and dynamic properties of a variety of bulk simple liquid metals $[3,4]$ and free liquid surfaces (FLS) [5].

\footnotetext{
*e-mail: beatriz@metodos.fam.cie.uva.es
} 
In 1981 Rice and co-workers predicted, from theoretical considerations and Monte Carlo simulations, that the atomic density near the free surface of a metallic liquid would change in a nonmonotonic way, showing a stratification in layers parallel to the interface [6]. The experimental confirmation of surface layering was first observed, several years later, in the x-ray reflectivity of liquid $\mathrm{Hg}$ [7]. Since then, the reflectivities of other five elemental liquid metal surfaces have been measured, namely, Ga, In, K, $\mathrm{Sn}$ and $\mathrm{Bi}$ [8], all of them displaying (or suggesting, in the case of $\mathrm{K}$ ) the expected layering peak. Moreover, $\mathrm{Sn}$ and $\mathrm{Bi}$ have shown a weak wide shoulder at a lower $q_{z}$ that is not present in the other systems. The data obtained for these liquid metals and several binary alloys [9] have shown that the FLS exhibits an oscillatory surfacenormal density profile (DP) which extends several atomic diameters into the bulk liquid. In these works the shape of the DPs were deduced by fitting the measured reflectivities to those predicted within a specific profile model, namely, the distorted crystal model, or variations thereof [8]. Although it would be desirable to obtain the DP in a model-independent way, the lack of phase information in the measurements renders this impossible.

In this paper we report a study of the surface structure of liquid Sn (1-Sn), performed through OF-AIMD simulations. Moreover, we have also analyzed the collective dynamics of the system at different depths from the FLS. To date, we are awere of only one experimental study of this topic using inelastic x-ray scattering in the FLS of indium [10]. This study showed that the effect of capillary waves is not only present on the surface, but also at very deep regions below the FLS. To our knowledge, the present paper reports the first ab initio study on the collective dynamics of a free liquid surface.

\section{Computational method.}

The total potential energy of $N$ ions with valence $Z$, enclosed in a volume $\Omega$, and interacting with $N_{e}=N Z$ valence electrons is written, within the Born-Oppenheimer approximation, as the sum of the direct ion-ion coulombic interaction energy and the ground state energy of the electronic system under the external potential created by the ions.

According to DFT, the ground state electronic density, $n_{g}(r)$, minimizes an energy functional given by the sum of the non-interacting electronic kinetic energy, $T_{s}[n]$, the electron-ion interaction energy, $E_{\text {ext }}[n]$, the classical electrostatic Hartree energy, $E_{H}[n]$, and the exchange-correlation energy, $E_{x c}[n]$, for which we have used the local density approximation, as parametrized by Perdew and Zunger [11]. Different expressions have been proposed for $T_{s}[n]$ and in this work we have used an average density model $[3,4]$.

The ion-electron interaction is described by means of pseudopotentials. In the OF-AIMD method the valence electron density is the basic variable, and non-local pseudopotentials cannot be used. The construction of the local pseudopotentials for our system has two steps. We start from an initial local pseudopotential proposed for Sn by Bhuiyan et al [12] which, in combination with the OF-AIMD method, has provided a good description of the static properties of liquid Sn at thermodynamic conditions near its triple point. However, the method used to construct the pseudopotential is not completely optimal, since it only tries to represent the static behavior but not the dynamical one, where the interaction between the different particles is taken into account at different time and length scales. A more realistic method should explicitly account for the presence of atoms around the central one and their effect on 
the force it feels. For this purpose, we have used a force matching method developed recently [13] to obtain a more accurate LPS. In figure 1 we have plotted the local pseudopotential before and after the force-matching process. We can observe how, in order to better represent the interactions, the pseudopotential has to adopt a higher value in reciprocal space near $q=0$. Using as benchmark the results of a KS-AIMD calculation for l-Sn [14], in figure 2 we have plotted the differences in the $x$ and $z$ components of the forces felt by the atoms as obtained in the OF and in the KS calculations at a particular liquid configuration before and after the force matching process. We can observe how with the initial local pseudopotential we can recover reasonably well the forces in the bulk region, but not at the surface (particles are sorted according to their $z$ coordinate). However, after the force matching process, these forces are recovered and the ones in the bulk are improved.

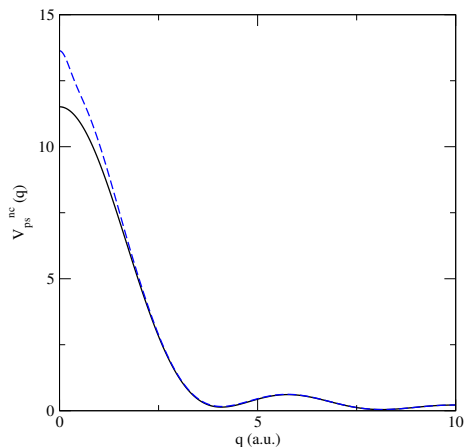

Figure 1. Non coulombic part of the local pseudopotential before (full curve) and after (dashed curve) force-matching.
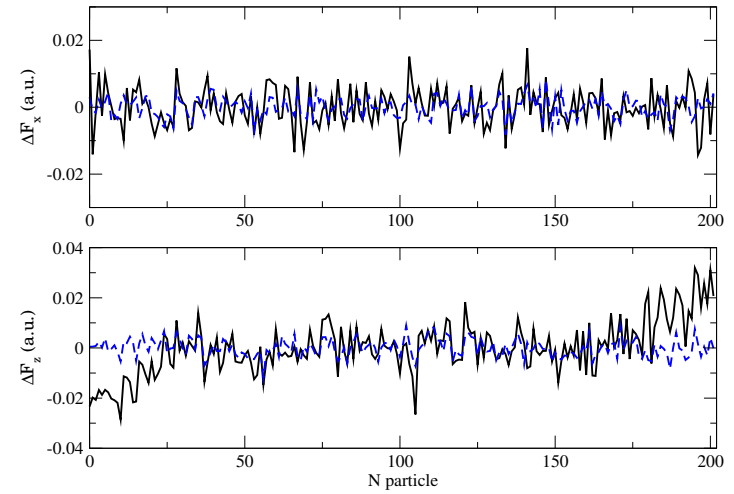

Figure 2. Difference between the $x$ and $z$ components of the forces as computed with KS-AIMD simulations [14] and OF-AIMD calculations, before (solid line) and after (dashed line) force-matching.

\section{Results and discussion.}

The simulated sample contains 2000 Sn atoms, enclosed in a tetragonal box with $L_{x}=L_{y}=34.25 \AA$, and $L_{z}=66.0 \AA$ in the case of $1000 \mathrm{~K}$. At the lower temperature of $600 \mathrm{~K}$, the dimensions of the tetragonal box are $L_{x}=L_{y}=33.80 \AA$, and $L_{z}=66.0$ $\AA$. In both cases the atoms formed a slab of initial width (approximately $50 \AA$ ) corresponding to the experimental number density $\left(0.035\right.$ and $0.337 \AA^{-3}$ at 600 and $1000 \mathrm{~K}$ respectively) with $8 \AA$ of vacuum at each side. Periodic boundary conditions are applied in all directions, meaning that each surface has a separation of $16 \AA$ with the adjacent image. This distance has proven to be enough for the different images not to interact with each other $[15,16]$. The system was first allowed to evolve at the desired temperature until the slab width accomodated the requirement of null pressure and equilibrium was reached, and after that, 20000 configurations were generated using a time step of $4 \mathrm{fs}$. These were used for the analysis of the properties of the slabs. The slab densities after equilibration were 0.0340 and 0.0332 $\AA^{-3}$ at 600 and $1000 \mathrm{~K}$, in good agreement with the experimental ones. 


\subsection{Static structure}

The averange ionic DP was computed from a histogram of the particle positions relative to the slab's center of mass, so that both halves of the slab are being averaged. The obtained results are shown in figure 3 , where we observe stratification for at least four layers into the bulk liquid. The spacing between the outermost layer and the first inner one is $\lambda_{\text {out }}=2.83 \AA$, whereas the inner interlayer distances are somewhat larger, namely, $\lambda_{\text {in }}=2.97 \AA$ at $1000 \mathrm{~K}$. It should be noted that the average DP obtained through the KS-AIMD [14] led to equal interlayer distances of $3.1 \AA$, which highlights the dependence of the average DP on the conditions of the simulation, in particular the lateral size of the box. At the lower temperature of $600 \mathrm{~K}$ the interlayer distances obtained are essentially all equal, with $\lambda_{\text {out }} \approx \lambda_{\text {in }}=2.86 \AA$.

In order to compute the reflectivity and compare with the experimental reported results, capillary waves have to be substracted from the system. The most convenient method is to obtain the intrinsic density profile through the calculation of the separation surface between the liquid and vapour phases, which is smoothed afterwards, following the procedure of Chacon and Tarazona [17]. In figure 3 we show the intrinsic DP along with the average DP for the two temperatures studied. The layered structure of the interface is more marked in the intrinsic profiles. Moreover, the obtained interlayer distances are modified from those in the average DP. The outermost interlayer distance is $\lambda_{\text {out }}=2.96 \AA$, with the inner interlayer distances being $\lambda_{\text {in }}=3.02 \AA$ at $1000 \mathrm{~K}$. At the lower temperature of $600 \mathrm{~K}$ the difference between the outermost and inner interlayer distances is more emphasized, with $\lambda_{\text {out }}=2.83 \AA$ and $\lambda_{\text {in }}=3.01 \AA$. It is important to note that, despite the differences between the average DPs obtained in this work and the one obtained in the KS-AIMD study, the corresponding intrinsic DPs practically coincide (see figure 3 ).

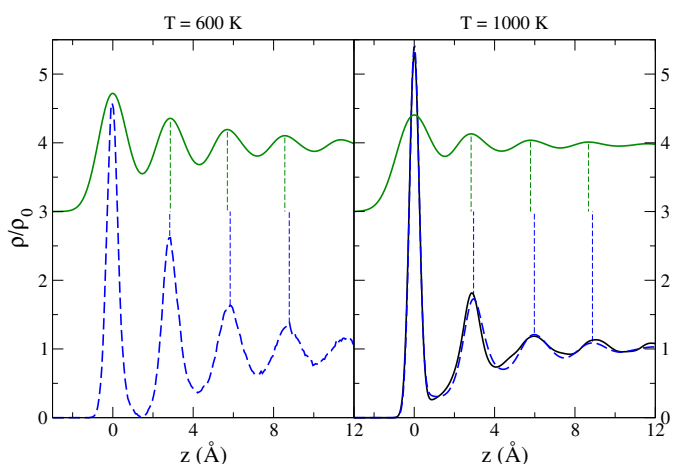

Figure 3. Average ionic DP (full curve, shifted upwards by 3 units) and intrinsic ionic DP (dashed curve) at $\mathrm{T}=600 \mathrm{~K}$ and $1000 \mathrm{~K}$. At 1000 $\mathrm{K}$ the lower full curve corresponds to the intrinsic ionic DP obtained using the simulation data from [14].

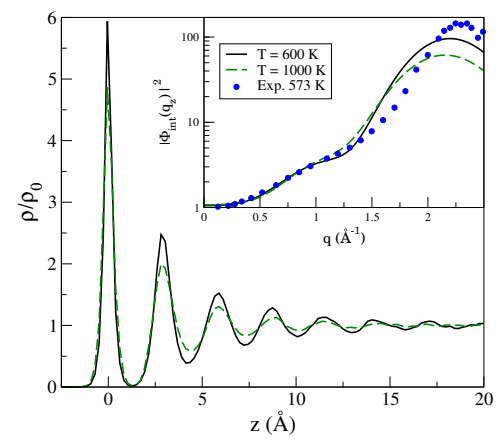

Figure 4. Comparison of the intrinsic electronic density profiles at $600 \mathrm{~K}$ (full line) and $1000 \mathrm{~K}$ (dashed line). Inset: comparison of reflectivities at $600 \mathrm{~K}$ (full line) and $1000 \mathrm{~K}$ (dashed line) with the experimental reflectivity (circles) [18].

The x-ray reflectivity, which is the property amenable to experimental observation, is related to the electronic DP. We have calculated the intrinsic electron DP, as obtained from a superposition of atomic electron densities centered at each ionic po- 
sition, using the smoothed separation surface. The intrinsic reflectivity is calculated as $R_{\text {int }}\left(q_{z}\right)=\left|\Phi_{\text {int }}\left(q_{z}\right)\right|^{2}$, where

$$
\Phi_{\mathrm{int}}\left(q_{z}\right)=\frac{1}{\rho_{e 0}} \int_{0}^{\infty} d z \exp \left[-i q_{z} z\right] \frac{d \rho_{e ; \text { int }}(z)}{d z}
$$

where $\rho_{e 0}$ is the average bulk electron density. The results obtained are shown in figure 4 , where we see a characteristic peak located around $q_{z} \simeq 2.2 \AA^{-1}$, which is close to $2 \pi / \lambda_{\text {in, } 600 \mathrm{~K}} \simeq 2.1 \AA^{-1}$, and is related to the layering of the DP. Morever, at around $q_{z} \simeq 0.8 \AA^{-1}$ we obtain a shoulder, more emphasized at $600 \mathrm{~K}$ than at $1000 \mathrm{~K}$, which also appears in the experimental measurements perfomed by Shpyrko et al [18]. The experimentalists ascribed the origin of the shoulder to an increased density in the outermost layer related to its smaller interlayer distance. However this interpretation may be biased by the model that was used and a deeper analysis would be valuable. This will be reported elsewhere.

\subsection{Dynamic properties}

We want to study the collective dynamics in the slab for different regions at different depths from the interface. To this end we define the collective properties (number of atoms, particle density, particle current) corresponding to a given region by multiplying the usual definition by a window function that is one inside the region and falls smoothly to zero outside.

From these we compute the corresponding time correlation functions. For instance, the intermediate scattering function, $F(q, t)$ is obtained as

$$
F(\vec{q}, t)=\left\langle\rho\left(-\vec{q}, t+t_{0}\right) \rho\left(\vec{q}, t_{0}\right)\right\rangle,
$$

and its FT into the frequency domain is the dynamic structure factor, $S(q, \omega)$.

The symmetry of the system defines a special direction, the $z$-axis, around which the properties must show cylindrical symmetry. We are particularly interested in waves that propagate parallel to the interface, and therefore among the $\vec{q}$ vectors allowed by the periodic boundary conditions, only those that are perperdicular to the $z$-axis have been used. In the averages like those of equation 2 we consider all vector orientations that fulfill this condition.

The calculated $S(q, \omega)$ presented in figure 5 at different depths in the slab show side-peaks, indicative of collective density excitations, up to $q \approx 1.23 \AA^{-1}$. Moreover, as one approaches the interface region coming from the bulk, the frequency of the side peaks increases, while the corresponding widths also increase, making the peaks less marked. From the position of the side peaks with respect to $q$ we can obtain the dispersion relation (DR) of the dynamic structure factor, depicted in figure 6 . Evaluating the slope of the curve for small $q$ it is possible to obtain an estimate of the adiabatic sound velocity at the corresponding layer. In this case we obtain an increase in the sound velocity from $2700 \pm 150 \mathrm{~m} / \mathrm{s}$ at the bulk region to $3600 \pm 200$ $\mathrm{m} / \mathrm{s}$ in the outermost layer. This increase suggests a higher ionic density and a lower compressibility in the outermost layer with respect to the bulk region.

We have also computed the longitudinal and transverse components of the current due to the overall motion of the particles. For the transverse current, two independent components appear due to symmetry, and here we are only considering the component associated to the motion of the particles along the $z$ axis. In figures 7 and 8 we have depicted the DR of the spectra of both currents. In the case of $\omega_{L}$, we clearly 


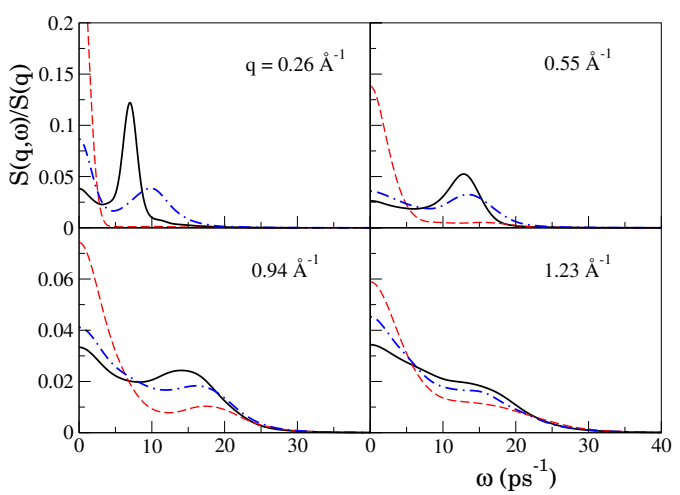

Figure 5. Dynamic structure factor at different $q$ values and depths: outmost layer (dashed curve), first inner layer (dot-dashed curve) and bulk region (full curve).

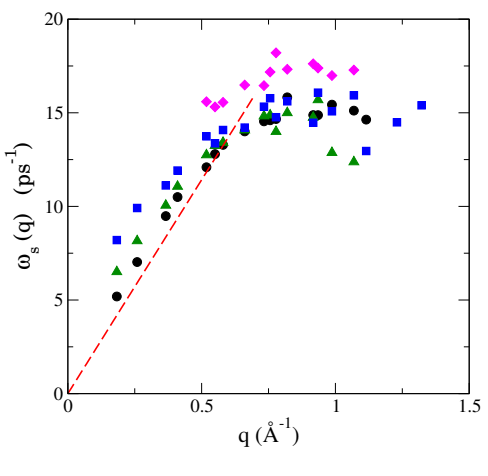

Figure 6. Dispersion relation of $S(q, \omega)$ at different depths: outmost layer (diamonds), first inner layer (squares), second inner layer (triangles), and bulk region (dots). Dashed curve: Experimental value of the adiabatic sound velocity at $1273 \mathrm{~K}$ [19] $(2461 \mathrm{~m} / \mathrm{s})$.

observe, apart from the usual sound propagation mode, a second contribution at lower frequencies that only appear at small $q$. This second contribution relates to the DR of capillary waves described by [8], $\omega \cong \sqrt{\gamma / \rho} q^{3 / 2}$. Note that the effect of the capillary waves not only appears in the first layers of the slab but extends very deep under the surface. This behaviour was previously observed in an experimental study of a liquid slab of In [10]. The transverse current in the $z$ direction in principle should contain contributions from capillary waves and from shear waves. The latter are only supported starting from a non-zero value of $q$, whereas the former should disperse as commented above in the small $q$ region. The results shown in figure 8 follow this behaviour for $q \rightarrow 0$, while for larger wavevectors it is not directly possible to disentangle the contribution of each type of waves. However, comparison with the bulk transverse DR, which has lower energy, suggests that we are observing the dispersion of the capillary waves moving from the hydrodynamic into the kinetic regime.

\section{Conclusions.}

We have been able to construct a LPS to study through OF-AIMD simulations the free liquid surface of Sn, using the force-matching method developed recently. Using this LPS, a liquid slab of $2000 \mathrm{Sn}$ atoms has been studied at $600 \mathrm{~K}$ and $1000 \mathrm{~K}$, obtaining very good results for the reflectivity as compared to experimental data, including the presence of the characteristic shoulder at $q \simeq 0.8 \AA^{-1}$. Due to the large number of particles, dynamic properties have also been studied in the liquid slab. We have obtained an increase in sound velocity of around $30 \%$ when going from the central region (behaving like a bulk) to the surface, suggesting a denser and less compressible surface as compared to the bulk. When studying the dispersion relation of longitudinal and transverse waves we obtain a second contribution, related to the presence of capillary waves, extending even to the central region of the slab. 


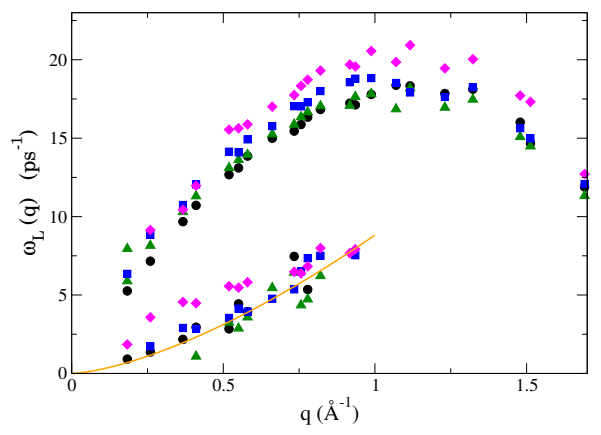

Figure 7. DRs of $C_{L}(q, \omega)$ at different depths: the symbols have the same meaning as in figure 6 . The higher frequencies correspond to sound propagation while the lower ones are related to capillary waves. Full curve: theoretical DR of capillary waves.

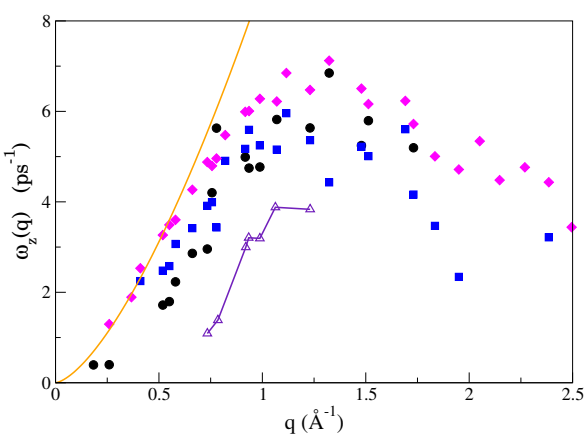

Figure 8. DR of $C_{T z}(q, \omega)$ at different depths: the symbols have the same meaning as in figure 6. Full curve: theoretical DR of capillary waves. Full curve with open triangles: DR from bulk 1-Sn.

We acknowledge the support of the MEC (FIS2014-59279-P) and JCyL (VA104A11-2). BGR acknowledges the financial support of Universidad de Valladolid.

\section{References}

[1] W. Kohn and L. J. Sham, Phys. Rev. 140, A1133 (1965).

[2] P. Hohenberg and W. Kohn, Phys. Rev. 136, B864 (1964).

[3] D. J. González et al, Phys. Rev. B 65, 184201 (2002).

[4] D. J. González et al, J. Chem. Phys. 115, 2373 (2001); L. E. González, D. J. González and J. M. López, J. Phys.: Condens. Matter 13, 7801 (2001); A. Delisle, D. J. González and M. J. Stott, Phys. Rev. B 73, 064202 (2006); L. E. González and D. J. González, Phys. Rev. E 77, 064202 (2008).

[5] D. J. González, L. E. González and M J Stott, Phys. Rev. Lett. 92, 085501 (2004); 94, 077801 (2005); Phys. Rev. B 74, 014207 (2006).

[6] M. P. D'Evelyn and S. A. Rice, Phys. Rev. Lett. 47, 1844 (1981).

[7] O. M. Magnussen et al, Phys. Rev. Lett. 74, 4444 (1995); E. DiMasi et al, Phys. Rev. B 58, R13419 (1998)

[8] P. S. Pershan and M. L. Schlossman, Liquid Surfaces and Interfaces. Synchrotron X-ray Methods (New York: Cambridge University Press, 2012).

[9] P. S. Pershan, Eur. Phys. J. - Special Topics, 196, 109 (2011).

[10] B. Wehinger, M. Krisch and H. Reichert, New J. Phys. 13, 023021 (2011).

[11] J. P. Perdew and A. Zunger, Phys. Rev. B 23, 5048 (1981).

[12] G. M. Bhuiyan, L. E. Gonzalez and D. J. Gonzalez, Eur. Phys. J. - Web of Conferences 15, 01011 (2011).

[13] B. G. del Rio and L. E. González, J. Phys.: Condens. Matter 26, 465102 (2014).

[14] L. Calderín, L. E. González and D. J. González, Phys. Rev. B 80, 115403 (2009). 
[15] D. J. González, L. E. González and M. J. Stott, Phys. Rev. Lett. 94, 077801 (2005).

[16] L. E. González, D. J. González and M. J. Stott, J. Chem. Phys. 123, 201101 (2005).

[17] E. Chacon and P. Tarazona, Phys. Rev. Lett. 91, 166103 (2003).

[18] O. G. Shpyrko et al, Phys. Rev. B 70, 224206 (2004).

[19] S. Blairs, Int. Mater. Rev. 52, 321 (2007). 